\title{
Los Sistemas de Educación Superior en Argentina y Bolivia - Estudio Comparado
}

\section{Lilian Constanza Diedrich ${ }^{\mathrm{a}}$, Alicia del Rosario Bassani ${ }^{\mathrm{b}}$ i Marco Antonio Limarino $^{c}$}

Rebut: 23/09/2014 Acceptat: 17/11/2014

\section{Resumen}

Se realiza un análisis comparativo de las políticas públicas de educación superior en Argentina y Bolivia.

Lo político se expresa a través de diferentes dimensiones: se establece entonces una comparación entre los aspectos normativos, la consecuente estructura organizativa del sistema de educación superior y la expansión del mismo en ambos países.

El análisis se realiza enfatizando la importancia del contexto en la determinación de las políticas, desde una perspectiva social, cultural, económica y política

El enfoque de educación comparada que se utiliza se centra en un abordaje desde la teoría del conflicto por considerársela superadora del enfoque racional, al abordar la realidad desde su multidimensionalidad.

El sistema de educación superior de la República Argentina es binario (educación universitaria y educación superior no universitaria); el del Estado Plurinacional de Bolivia comprende en su estructura cuatro niveles (Formación de Maestras y Maestros; Técnica y Tecnológica; Artística y Universitaria).

En el caso argentino la ley que lo normaliza es del año 1994 (pese a que el contexto político actual presenta notables diferencias con el año de sanción de la misma); en Bolivia se sanciona en el año 2010 la Ley 070, que representa los valores del grupo en ejercicio del poder actual.

Palabras claves: educación superior- política-estructura-normas

\footnotetext{
a Universidad Nacional de Salta. Salta - Argentina

${ }^{b}$ Universidad Nacional de Salta. Salta - Argentina

c Universidad Católica Boliviana San Pablo-Bolivia
} 


\section{Higher Education System in Argentina and Bolivia: a Comparative Analysis}

\section{Abstract}

We performed a comparative analysis of public policies for higher education in Argentina and Bolivia.

The policies are expressed through different dimensions: the normative aspects, the consequent organizational structure of the higher education system, and its expansion in both countries.

The comparative analysis is done by emphasizing the importance of the context in determining the policies from a social, cultural, economic, and political perspective.

The used comparative education is approached from the theory of conflict, which is recognized as going beyond the rational approach since the former addresses the reality from its multidimensionality.

The higher education system of the Argentina Republic is binary (university education and non-university higher education); the one corresponding to the Plurinational State of Bolivia comprises four levels in its structure (Teacher Training, Technical and Technological, Artistic, and University).

In the Argentine case, the law normalizing the education system is from 1994 (although the current political context differs markedly with that of 1994). In the case of Bolivia the law 070 is from 2010, which represents the values of the group in exercise of the current power.

Keywords: higher education-policy-framework-rules

\section{Introducción}

El presente trabajo realiza una comparación de los subsistemas de educación superior en Argentina y Bolivia, identificando semejanzas y diferencias para contribuir a mejorar el conocimiento mutuo.

Dada la cercanía geográfica de los países, el flujo de intercambio entre ellos es importante, existiendo una numerosa comunidad de residentes bolivianos en Argentina. 
Específicamente el norte tiene una fuerte relación con Bolivia; la cantidad de estudiantes de universidades argentinas provenientes de Bolivia es significativa en las provincias de Salta y Jujuy.

Existen experiencias de reconocimiento de estudios entre universidades de un país y otro a través de convenios específicos entre las instituciones, por lo general de carácter privado.

Aunque recientemente ambos países firmaron un convenio bilateral de reconocimiento de estudios superiores, debe avanzarse en la determinación de los mecanismos y procedimientos para la efectivización del mismo.

No podemos desconocer que los aspectos culturales y demográficos cumplen un papel fundamental en la comprensión de las semejanzas y diferencias en la conformación de los sistemas de educación superior.

Uno de los fenómenos sociales de mayor relieve en la América Latina de finales del siglo XX es la emergencia de los pueblos indios. En los últimos años se ha producido un crecimiento rápido y dinámico de las numerosas organizaciones indígenas que, desde un amplio abanico de posiciones ideológicas y de modo cada vez más coordinado, han confluido en un amplio movimiento de dimensiones generales, exigen la autodeterminación de los pueblos y la redefinición del Estado como Estado multiétnico y pluricultural

En Bolivia, por lo menos el $60 \%$ de la población habla una lengua originaria, sea en condición de monolingües (12\%) o de bilingües (48\%). Son 36 los pueblos indígenas que coexisten en el territorio del actual Estado boliviano aunque Bolivia es generalmente considerada como un país eminentemente andino y altiplánico, y, por ende, quechua y aimara, cuando en rigor la mayor parte del territorio boliviano pertenece a lo que se denomina Oriente, Chaco y Amazonía, espacio caracterizado también por la mayor diversidad étnico-cultural y lingüística.

Los pueblos indígenas de los Andes bolivianos, es decir, la zona occidental o andina del país son tres: aimaras, quechuas y urus. Los quechuas históricamente se han ubicado en los valles interandinos y los aimaras y urus en las zonas altiplánicas. Los miembros de pueblos indígenas andinos comprenden aproximadamente entre el $40 \mathrm{y}$ el $90 \%$ de la población que habita esa región, dependiendo de su ubicación en áreas rurales o urbanas; ellos constituyen el alrededor del $60 \%$ de la población total del país.

Sin perder de vista la complejidad y multidimensionalidad de la conformación de los sistemas educativos, centraremos el análisis en los siguientes ejes: estructura, legislación que los regula, existencia de organismos de acreditación y dimensión numérica. 


\section{Estructura}

\section{Argentina}

En el sistema educativo argentino la educación superior es impartida por las universidades, los institutos universitarios y los institutos superiores no universitarios. Sólo las instituciones ubicadas en alguna de estas tres categorías están legalmente autorizadas por el Ministerio de Educación de la Nación para otorgar títulos oficiales. La diferencia entre las dos primeras categorías consiste en que los institutos universitarios ofrecen carreras de un sólo campo de conocimiento mientras que las universidades comprenden distintos departamentos cubriendo distintas ramas de la formación superior. Las universidades y los institutos universitarios otorgan títulos de grado (licenciaturas, ingenierías, etcétera) y postgrado (especializaciones, maestrías y doctorados), y en algunos casos también pregrado, como títulos intermedios para quienes están cursando carreras de grado, o bien directamente como carreras cortas enfocadas en la práctica laboral en algunas disciplinas (funcionando en ese caso también como institución terciaria). Por su parte los institutos no universitarios sólo ofrecen carreras cortas llamadas tecnicaturas (2 a 4 años de duración), consistentes en títulos de pre-grado que brindan a los estudiantes las herramientas necesarias para insertarse en la actividad laboral en empleos con calificación especializada; los institutos de formación docente ofrecen la carrera de profesorado.

- Universidades: desarrollan su actividad en una diversidad de áreas disciplinarias no afines, organizadas en facultades, departamentos o unidades académicas equivalentes (art.26-Ley 24.521).

- Institutos Universitarios: tienen las mismas características de funcionamiento que las universidades pero circunscriben su actividad académica a una sola área disciplinaria.

- Instituciones de Nivel Superior No Universitario: son instituciones que desarrollan la formación docente y/o de carácter instrumental en las áreas humanística, social, técnico-profesional y artística.

- Colegios Universitarios: son instituciones de nivel superior no universitario que tienen acuerdos de intercambio académico con universidades (art. 22Ley 24521).

Los estudiantes que completan sus estudios en los Institutos de Educación Superior no Universitarios (IESNU) pueden continuar sus estudios en la universidad mediante lo que se conoce como articulación, para obtener el título universitario de grado correspondiente a su profesión. 


\section{Bolivia}

La educación superior de formación profesional en Bolivia comprende las siguientes categorías: Formación de Maestros y Maestras, Formación Técnica y tecnológica, Formación Artística y Formación Universitaria

\section{Formación de Maestras y Maestros}

La Formación Inicial se desarrolla a través de las Escuelas Superiores de Formación de Maestras y Maestros.

El Ministerio de Educación otorga el Título de Maestro con grado de Licenciatura y su respectivo reconocimiento en el Escalafón del Magisterio.

La Formación Post gradual se desarrolla por la Universidad Pedagógica, con dependencia del Ministerio de Educación.

\section{Formación Técnica y Tecnológica}

La Estructura Institucional de la Formación Superior Técnica y Tecnológica está constituida por:

-Institutos Técnicos e Institutos Tecnológicos, que desarrollan programas de formación profesional a nivel técnico

-Escuelas Superiores Tecnológicas, que desarrollan programas complementarios de formación especializada a nivel licenciatura para profesionales del nivel técnico superior

\section{Formación Artística}

La estructura institucional de la Formación Artística está constituida por:

-Centros de Capacitación Artística, espacios educativos que desarrollan programas de corta duración

-Institutos de Formación Artística, espacios educativos que desarrollan programas de formación artística a nivel técnico medio y superior. Los niveles son capacitación, técnico medio y técnico superior.

-Escuelas Bolivianas Interculturales que desarrollan programas especializados de formación profesional a nivel licenciatura en las diferentes expresiones artísticas. El nivel que otorga es el de licenciatura. 


\section{Formación Universitaria}

Los niveles y grados académicos reconocidos son:

1. Pre grado:
a) Técnico Superior.
b) Licenciatura.

2. Post grado:
a) Diplomado.
b) Especialidad.
c) Maestría.
d) Doctorado.
e) Post doctorado

Las Universidades reconocidas por el Estado Plurinacional de Bolivia son:
a) Universidades Públicas Autónomas.
b) Universidades Privadas.
c) Universidades Indígenas.
d) Universidades de Régimen Especial en las que se encuentran la Universidad Militar y la Universidad Policial.

Podemos observar un desarrollo académico institucional paralelo, al considerar que la formación de maestros, la formación técnica y tecnológica y la formación artística, todas ellas conducen hasta el nivel de licenciatura y pueden incluso llegar hasta los postgrados.

\section{Normativa}

\section{Argentina}

El sistema de educación superior se encuentra reglamentado por la ley 24521 sancionada en julio de 1995.

La ley por primera vez intentó generar un sistema de educación superior, incluyendo universidades y entidades que si bien se inscriben en un nivel terciario de educación, 
no asumen la forma clásica y tradicional de las universidades. Allí están incluidos los Institutos de Formación Docente, los Terciarios Técnicos y los de Formación Artística. Comparten esas áreas tanto las entidades de gestión pública como de gestión privada.

Esta norma legal trata en pié de igualdad a las Universidades públicas y privadas y regula orgánicamente todas las instituciones del Nivel Superior, universitarias y no universitarias, nacionales, provinciales y municipales, estatales y privadas (art.1). Específicamente prevé la articulación entre los distintos tipos de instituciones que integran el nivel.

Otro organismo creado por la nueva ley de Educación Superior es el llamado Consejo de Universidades. Es el ámbito donde las Universidades de gestión estatal o privada, y los denominados Consejos de Planificación Regional, se reúnen periódicamente para actuar como un Parlamento de Universidades. Lo hacen en el ámbito físico y con la conducción del Ministerio de Educación

Es el espacio natural para el esclarecimiento y concertación que tiene el Estado Nacional y las Universidades, para jerarquizar a las Universidades y estimular la vida académica de ellas. Su funcionamiento, a pesar de algunos períodos discontinuos, ha demostrado claras ventajas y podríamos señalar que por primera vez en casi 45 años, ha constituido el hecho más plausible de una real política de Estado para las Universidades Argentinas.

\section{Bolivia}

La educación superior está regida por la Constitución Política del Estado (CPE) sancionada en 2009, la Ley de Educación denominada Avelino Siñani - Elizardo Pérez y los reglamentos sobre diversos aspectos y componentes de la estructura educacional que aún se encuentran en proceso de elaboración, y unos pocos se encuentran ya elaborados y en vigencia.

La LEY NRO 070/2010 "AVELINO SIÑANI - ELIZARDO PÉREZ", legisla todo el sistema educativo plurinacional. No existe una normativa diferenciada para el nivel superior.

La Constitución Política del estado en su, articulo 91, dice: "La educación superior es intercultural y plurilingüe, y tiene por misión la formación integral de recursos Humanos con alta calidad y competencia profesional".

Consagra siete artículos (Arts. 91 al 97) a la educación superior, de los cuales queremos destacar algunos aspectos:

Un primer punto por señalar es que la universidad se define como una instancia de formación profesional, de producción y divulgación de conocimientos, donde la novedad es la consideración de conocimientos y saberes indígenas y campesinas con relación al conocimiento científico; lo cual es un desafío de políticas de conocimiento 
en las universidades y sus respectivos diseños curriculares, al menos si éstas se encuentran sensibles a estas temáticas.

En este mismo sentido, la Nueva Ley de Educación Avelino Siñani y Elizardo Perez (promulgada en 2006), proclama la interculturalidad e intraculturalidad, la integración de saberes y la descolonización del conocimiento como ejes de las políticas universitarias bolivianas.

Pese a no haber una aplicación concreta de la Ley, el cambio social y el peso discursivo produjo impactos internos en las universidades. Pueden observarse cambios en el nivel institucional, con intentos de reforma y de creación de nuevas modalidades de atención a las poblaciones indígenas bajo la forma de programas especiales, universidades paralelas o facilidades de acceso y becas sindicales.

Dicha Ley, totalmente carente de lineamientos o dispositivos para su puesta en práctica, simplemente profundizó el quiebre de la universidad con el Estado, ahondando la brecha entre dos universos sin lenguaje común ni espacio de diálogo, abriendo un nuevo frente de competición para las Universidades públicas: las nuevas universidades indígenas promovidas por el Estado (Weise,2012)

En sus artículos 92 y 93, la CPE, deja intacta la autonomía universitaria y el financiamiento. Este último artículo plantea no obstante otros desafíos para las universidades públicas como la creación de "mecanismos de participación social de carácter consultivo, de coordinación y asesoramiento" o el establecimiento de programas de desconcentración académica y de interculturalidad que consideren las necesidades de los pueblos indígenas originario campesinos o, en fin, la participación en la creación de universidades comunitarias y pluriculturales en áreas rurales. Deja establecido que la universidad determinará los mecanismos de rendición de cuentas y de transparencia.

Otro desafío que emerge de la CPE es la creación de centros interculturales de formación y capacitación técnica, de libre acceso al pueblo, así como la implementación de programas de recuperación y desarrollo de las diferentes lenguas de las naciones y pueblos indígenas originario campesinos (art. 95).

A nivel de las prácticas docentes, observamos un proceso de pérdida de identidad de los profesores, que ejercen su labor con una fuerte sensación de frustración e impotencia frente a los requerimientos de atención a la diversidad sociocultural, una vez que las identidades indígenas se han hecho presentes y se han visibilizado en las aulas universitarias. Las contradicciones se acentúan y se ponen de manifiesto, así como la carencia de recursos de los profesores para hacerles frente (Weise, 2011).

El artículo 92 de la Constitución Política del Estado Boliviano determina qué las "universidades públicas constituirán en ejercicio de su Autonomía la UNIVERSIDAD BOLIVIANA ", la que coordinará y programará sus fines y funciones mediante un organismo central de acuerdo a un Plan Nacional de Desarrollo Universitario. Es así que la Universidad Boliviana determinó constituir el Comité Ejecutivo de la 
Universidad Boliviana; donde su naturaleza jurídica se desprende del Artículo 92 de la $\mathrm{CPE}$, teniendo como fuente de financiación al Tesoro General de la Nación.

El Comité Ejecutivo de la Universidad Boliviana, es el organismo ejecutivo de coordinación, planificación y de programación de las actividades que realiza la Universidad Boliviana, su labor está enmarcada de acuerdo a las decisiones de los Congresos y Conferencias Nacionales de Universidades y a las gestiones encomendadas por las Casas de Estudios Superiores; además cumple funciones de representación especialmente ante los poderes del Estado.

Entre los servicios nacionales de importancia, que realiza el CEUB, están los de promover el intercambio de investigadores, el fomento de convenios entre las distintas universidades e instituciones nacionales e internacionales que pueden aportar a la educación del Sistema Universitario Boliviano.

Las universidades privadas tienen su propia ley y reglamentación específica. Decreto Supremo $N^{\circ}$ 1433, 12 de diciembre de 2012, en cuyo anexo se incorpora el reglamento de universidades privadas

La Asociación Nacional de Universidades Privadas es quién coordina a las universidades privadas en Bolivia, siempre y cuando se encuentren asociadas a esta entidad.

\section{Organismos de acreditacion de carreras}

\section{Argentina}

La evaluación y la acreditación de la calidad se han instalado fuertemente en la agenda de la educación superior regional

Estos procesos se han desarrollado en casi todos los países y se han constituido en herramientas útiles para superar gradualmente los problemas de diversificación organizacional, heterogeneidad de la calidad y crecimiento inorgánico del número de instituciones.(Fernandez,Lamarra, N-2006)

Para la evaluación de las Universidades e Institutos Universitarios crea la Comisión Nacional de Evaluación y Acreditación Universitaria (CO.N.E.A.U.), organismo descentralizado que desarrolla su actividad en la jurisdicción del Ministerio de Educación, cuyas funciones principales son: coordinar la evaluación externa de las universidades, acreditar las carreras de grado y posgrado, pronunciarse sobre la viabilidad del proyecto institucional y prepara informes sobre los pedidos de nuevas universidades.

Sus funciones han sido determinadas en el artículo 46 de la Ley 24.521 de Educación Superior de 1995. Es el único organismo público nacional de evaluación y acreditación universitaria y comenzó a funcionar en 1996. 
Las universidades deben someter sus planes de estudio a la CONEAU (Comisión Nacional de Evaluación y Acreditación Universitaria) como requisito para que los títulos que expiden tengan validez oficial.

Hasta ahora, la CONEAU cumplió un mandato, el que tiene que ser evaluado periódicamente. La ley no podía prever en ese entonces que la misión evaluadora de las Universidades asumiría una complejísima trama de trabajo que desbordaría cualquier estimación para las instituciones y para dicha Agencia. Esto es lo que ha ocurrido. Junto al proceso, puesto en movimiento, no ha existido tiempo material para perfeccionar buena parte de los procedimientos que producen estas nuevas experiencias. Se han mejorado algunos, pero la dinámica de los tiempos exige constantes cambios como es sabido. El proceso de acreditaciones en posgrado no es voluntario, es obligatorio. En el grado, las carreras que gradualmente se están incorporando al conocido art.43 de la ley es imperativo.

\section{Bolivia}

La ley 70/2010 crea Agencia Plurinacional de Evaluación y Acreditación de la Educación Superior Universitaria - (APEAESU). Con carácter descentralizado, conformado por un Directorio y un Equipo Técnico Especializado. Su funcionamiento y financiamiento será reglamentado por Decreto Supremo.

El Gobierno aprobó el Decreto 1433 que reglamenta el sistema universitario privado. Entre los artículos más importantes está la disposición de que las universidades privadas deben entregar al Estado el 1 por ciento del total de sus ingresos a favor de la Agencia de Acreditación del Estado. La Asociación de Universidades Privadas ya hizo una representación para ser escuchada, pues considera que una agencia de esta naturaleza "debe ser un órgano técnico que debe sustentarse con los aranceles de los servicios que presta"

Las universidades privadas funcionan en Bolivia hace tres décadas y actualmente cuentan con 150.000estudiantes.

Los rectores reconocen la importancia y necesidad de la acreditación, por lo que encaran "procesos internacionales de acreditación universitaria para llegar a estándares académicos en educación superior, que no tengan nada que envidiar a las universidades públicas".

En los hechos, pese a que con anterioridad se aprobaron dos veces, en 1994 y en 2005, leyes creando un organismo de acreditación, nunca fue puesto en marcha, resultado de una mezcla entre resistencias técnicas y políticas de instituciones públicas y privadas, y la escasa voluntad estatal. Sólo las universidades autónomas cuentan con su propio organismo de acreditación

Diversas universidades, públicas y privadas bolivianas, participan sin embargo de procesos internacionales de certificación, dentro "El Sistema de Acreditación Regional 
de Carreras Universitarias para el Mercosur (Arcu Sur)", un acuerdo suscrito entre los país miembros de Mercosur, Bolivia y Chile, como asociados.

La Agencia Plurinacional de Evaluación y Acreditación de la Educación Superior Universitaria - APEASU a la fecha no entró en vigencia.

Su implementación se encuentra trabada debido a situaciones de orden político al considerar bajo presión sectorial, la inclusión de dos representantes del sector de formación de docentes. Al ser este un sector donde el gremio es relativamente fuerte, las negociaciones en relación a la cuota de poder se encuentran momentáneamente suspendidas.

Desde la perspectiva de la constitución política, la intromisión del sector docente es inconstitucional. Así también desde la perspectiva de la independencia que indica la constitución al insistir el gobierno la representación en el directorio de al menos 2 representantes cuando la constitución indica que esta entidad debe ser independiente.

Actualmente es CEUB quién se ocupa del proceso de evaluación y acreditación de programas académicos, tal como se hace desde hace varios años. La entidad, entre otros, fue creada para esos fines.

\section{Los sistemas en cifras}

El sistema Univ. Boliviano está compuesto por 58 universidades (De la CEUB 12, privadas 39, del régimen especial 4 y 3 universidades indígenas). Estas 58 universidades están regidas bajo leyes y estamento universitarios

Las universidades públicas en Bolivia ofrecen cerca de 250 programas académicos en seis áreas distintas, particularmente, economía y legislación, sociales y humanidades, ciencias naturales y biología, ciencias de la salud, técnicas en ingeniería y agricultura. Además de las instituciones financiadas por el estado, existen 33 instituciones privadas de educación superior en Bolivia, que ofrecen alrededor de 220 programas académicos.

En el año 2010 se matricularon 334.238 alumnos en las universidades públicas y en las privadas 94.748 haciendo un total de 429.986 matriculados.

El sistema universitario argentino está conformado por: 47 Universidades Nacionales, 49 Universidades Privadas,7 Institutos Universitarios Estatales, 14 Institutos Universitarios Privados,1 Universidad Provincial, 1 Universidad Extranjera y 1 Universidad Internacional

Al año 2010 el sistema universitario argentino contaba con un total de 1.718 .610 estudiantes de los cuales 1.366 .237 pertenecían a la universidades públicas y 352.270 a las universidades privadas. 


\section{Reconocimiento de titulos entre ambos paises}

En el año 2013 la Cámara de Diputados de Bolivia aprobó el proyecto de ley que ratifica el Convenio de Reconocimiento Mutuo de Títulos y Grados Académicos de Educación Superior entre la República Argentina y el Estado Plurinacional de Bolivia, suscrito en la ciudad de Cochabamba, el 18 de julio de 2012.

El objeto del proyecto es reconocer y conceder validez a los títulos y grados académicos de educación superior otorgados por las instituciones de Educación Superior reconocidas oficialmente por los sistemas educativos de cada Estado, a través de los respectivos organismos oficiales.

La ejecución del Convenio estará a cargo del Ministerio de Educación y del Comité Ejecutivo de la Universidad Boliviana (CEUB), por el Estado Plurinacional de Bolivia y، el Ministerio de Educación de la República Argentina.

El convenio prevé la constitución de una Comisión Bilateral Técnica conformada por representantes de las instancias competentes de ambos Estados, para la elaboración de una Tabla de Equivalencias y Acreditaciones.

\section{La situacion de las universidades indígenas}

En la introducción se había hecho referencia a los rasgos especiales de la conformación de la población en Bolivia: el 60 \% de la población del país pertenece a pueblos indígenas andinos.

El año 2008, el Presidente en ejercicio de Bolivia, Evo Morales Ayma, firmó el Decreto Supremo 29664, UNIBOL, que dispone la creación de tres "universidades indígenas" en los departamentos de La Paz, Cochabamba y Chuquisaca.

Desde el gobierno central se justifica la creación de estas universidades en la necesidad de crear otras opciones y oportunidades de estudio para sectores que históricamente fueron discriminados y en que estas universidades recuperan los saberes de los pueblos indígenas y complementan la enseñanza con los saberes de otros pueblos.

Están orientadas a la formación profesional de jóvenes miembros de de los llamados pueblos originarios indígenas y de campesinos quechuas (el quechua es una de las lenguas aborígenes sudamericanas.

La mirada está puesta en el desarrollo productivo comunal, municipal, departamental y nacional.

Una característica de UNIBOL es que se enseña en idioma nativo: quechua (Cochabamba), aymara (La Paz) y guaraní (Chuquisaca). El castellano no es desconocido, pero es una materia más, casi en el mismo plano que una lengua extranjera como el inglés o el francés. Asimismo, es un requisito que la tesis de grado sea defendida oralmente en el idioma nativo. 
En la universidad aymara se puede cursar agronomía altiplánica, industria de alimentos y textiles, veterinaria y zootecnia. En la quechua se imparte industria de alimentos, forestación y piscicultura. La universidad guaraní cuenta con industria hidrocarburífera, forestación, piscicultura, veterinaria y zootecnia. En calidad de transversales, se imparten materias de ciencias sociales, economía, historia y medio ambiente, entre otras, como deportes y artes. Esta selección fue hecha pensando en los especialistas que cada región necesita para aumentar la productividad de aquellas zonas.

El ingreso a estas universidades se hace sobre la base de un selección realizada por los líderes comunitarios y los alumnos viven como internos. Los egresados deben crear un proyecto para su comunidad, a la que deben regresar una vez obtenida la titulación.

La creación de estas universidades ha levantado muchas voces críticas cuestionando su existencia cuando en Bolivia hay universidades públicas y privadas a las que pueden asistir todos los bolivianos.

Consideran que la creación de estos centros responde a un discurso etnicista e ideologizado.

En el año 2014 se graduarán los primeros licenciados de las tres universidades indígenas del país. Alrededor de 172 estudiantes se encuentran en proceso de defensa de tesis, en idioma originario para obtener su título académico.

\begin{tabular}{|c|l|l|}
\hline Ítems & \multicolumn{1}{|c|}{$\begin{array}{c}\text { Sistema de Educación Superior } \\
\text { Argentina }\end{array}$} & $\begin{array}{c}\text { Sistema de Educación Superior } \\
\text { Bolivia }\end{array}$ \\
\hline Estructura & $\begin{array}{l}\text { Nivel Superior Universitario } \\
\text { Nivel Superior no Universitario }\end{array}$ & $\begin{array}{l}\text { Nivel Superior Universitario } \\
\text { Nivel Superior de Formación } \\
\text { profesional }\end{array}$ \\
\hline Normativa & $\begin{array}{l}\text { Ley 24.521 } \\
\text {-Idéntica para universidades públicas } \\
\text { yprivadas }\end{array}$ & $\begin{array}{l}\text { Ley 70/2010 } \\
\text { Constitución Política del Estado } \\
\text {-Diferente para universidades } \\
\text { públicas y privadas }\end{array}$ \\
\hline $\begin{array}{c}\text { Requisitos de } \\
\text { ingreso }\end{array}$ & $\begin{array}{l}\text {-Título de nivel medio } \\
\text {-Algunas requieren examen de } \\
\text { competencias }\end{array}$ & $\begin{array}{l}\text {-Título de nivel medio } \\
\text {-Algunas requieren examen de } \\
\text { competencias }\end{array}$ \\
\hline $\begin{array}{c}\text { Cantidad de } \\
\text { instituciones y } \\
\text { alumnos }\end{array}$ & $\begin{array}{l}\text { Un Universidades Nacionales, 49 } \\
\text { Universitarios Estatales,14 Institutos } \\
\text { Universitarios Privados,1 Universidad } \\
\text { Provincial, 1 Universidad Extranjera y y }\end{array}$ & $\begin{array}{l}\text { 58 universidades : de la CEUB 12, } \\
\text { universidades privadas 39, } \\
\text { universidades del régimen } \\
\text { especial 4 y 3 universidades } \\
\text { indígenas }\end{array}$ \\
\hline
\end{tabular}




\begin{tabular}{|c|l|l|}
\hline & $\begin{array}{l}1 \text { Universidad Internacional } \\
\begin{array}{c}1.718 .610 \text { de estudiantes: 1.366.237 } \\
\text { en universidades públicas y 352.270 } \\
\text { en universidades privadas }\end{array}\end{array}$ & $\begin{array}{l}\text { En el año 2010 se matricularon } \\
334.238 \text { alumnos en las } \\
\text { universidades públicas y en las } \\
\text { privadas 94.748 haciendo un total } \\
\text { de } 429.986 \text { matriculados. }\end{array}$ \\
\hline $\begin{array}{c}\text { Sistema de } \\
\text { Acreditación }\end{array}$ & $\begin{array}{l}\text { CONEAU-Comisión Nacional de } \\
\text { Evaluación y Acreditación } \\
\text { Universitaria }\end{array}$ & $\begin{array}{l}\text { APEASU-Agencia Plurinacional de } \\
\text { Evaluación y Acreditación del } \\
\text { Sistema de Educación Superior }\end{array}$ \\
\hline
\end{tabular}

Fuente: Elaboración propia

\section{Bibliografía}

ANUARIO DE ESTADISTICAS UNIVERSITARIAS (2010) -Secretaría de Políticas universitarias- República Argentina

Estado Plurinacional de Bolivia / Ministerio de Educación (2010). Ley de la Educación Avelino Siñani y Elizardo Pérez, La Paz, 20 de diciembre de 2010.

GARCÍA GARRIDO, J. L.: (1996) Fundamentos de Educación Comparada. Dykinson, 3.a ed., 1.a reimp., Madrid

FERRER, F. (1990) Educación Comparada: Fundamentos teóricos, metodología y modelos. PPU, Barcelona

FERNANDEZ LAMARRA (2007) Educación Superior y Calidad en América Latina y Argentina. Los procesos de evaluación y acreditación, EDUNTREF / IESALC-UNESCO, Buenos Aires

IESALC (2006) Informe sobre la educación superior en América Latina 2000-2005-La metamorfosis de la Educación Superior; Instituto Internacional de la UNESCO para la Educación Superior en América Latina y el Caribe (IESALC)

INEB (2012) Instituto Nacional de Estadísticas de Bolivia

RAMA, CLAUDIO (2006)-La Tercera Reforma de la Educación Superior en América Latina-Fondo de Cultura Económico-Argentina

República Argentina (1995) -Ley 24.521-Educacion Superior

República de Bolivia (2008)-La Constitución Política del Estado. La Paz.

REVILLA ORIAS, PAOLA (2011)- Universitario indígena, universitario boliviano: El proyecto descolonizador del Decreto 29664 de Evo Morales Ayma-Revista Isses No 9-

WEISE, CRISTA: (2012) Contribuciones para pensar las universidades en países con alta diversidad sociocultural, desde la experiencia boliviana- Propuesta Educativa Número 38 - Año 21 -Vol 2 - Págs 27 a 31 
WILSON MERCADO: El resurgimiento de las etnias en Bolivia y su influencia en la integración

YAPU, MARIO: (2011) Diversificación del campo de la educación Superior y las Universidades, La Paz, 2011, Instituto de Estudios Avanzados en Desarrollo, Serie de Documentos de Trabajo sobre desarrollo N²/2011

ZABALZA, MIGUEL ANGEL (2007) La enseñanza universitaria. El escenario y sus protagonistas. Narcea. Madrid

\section{Nota biográfica}

Lilian Constanza Diedrich. Grado Académico: Magíster en Nuevas Tecnologías Aplicadas a la Educación- Universidad Carlos III de Madrid-Universidad Autónoma de Barcelona-Universidad de Alicante

Secretaria Académica de la Facultad de Economía y Administración-Universidad Católica de Salta-Argentina-

Filiación Institucional: Universidad Nacional de Salta-Argentina

Alicia del Rosario Bassani. Grado Académico: Dra. En Investigación e Innovaciones Educativas. Universidad de Rovira i Virgili. Tarragona. España.

Profesora Asociada Regular -Cátedra Fundamentos de la Práctica Educativa-Facultad de Ciencias de la Salud- Universidad Nacional de Salta-Argentina

Filiación Institucional: Universidad Nacional de Salta-Argentina

Marco Antonio Limarino Cazón. Grado Académico: Magister en Administración de Negocios-Universidad Católica de Salta

Rector de la Sede Regional Tarija de la Universidad Católica Boliviana San PabloBolivia

Filiación Institucional: Universidad Católica Boliviana San Pablo-Bolivia 